\title{
Association of an ataxia indistinguishable from Friedreich's ataxia and congenital glaucoma in a family: a new syndrome
}

\author{
ONOFRE COMBARROS, JESÚS CALLEJA*, CARLOS LENO, AND \\ JOSÉ BERCIANO
}

From the Departments of Medicine (Section of Neurology) and *Clinical Neurophysiology, National Hospi Marqués de Valdecilla, Faculty of Medicine, Santander, Spain.

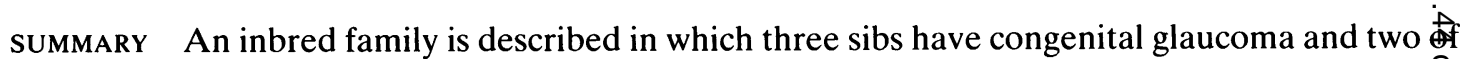
them also have an ataxia indistinguishable from Friedreich's ataxia. The association between these two disorders has not previously been reported. The genetic mechanisms of this association are discussed.

Impairment of vision often occurs in patients with spinocerebellar degenerations. The most frequent ocular disorders are optic atrophy or pigmentary retinal degeneration, but instances of cataract have also been described. ${ }^{1}$ Congenital glaucoma is not known to be associated with the hereditary ataxias. We report on three sibs, the offspring of consanguineous parents, who had congenital glaucoma in conjunction with an ataxia indistinguishable from Friedreich's ataxia in two cases.

\section{Case reports}

The pedigree of the family showed obvious consanguinity between the parents of the affected subjects (fig 1). The parents of our patients were examined and found to be normal. Four subjects in this family were affected by history. There is good evidence that all four had progressive ataxia in their first or second decade of life and severe visual failure in infancy. The paternal grandmother and the parents of the proband were convinced that the clinical picture of the affected members by history was the same as described below.

CASE VI.6

The proband was an 18 year old man whose motor development was normal until the age of four when progressive imbalance of gait and clumsiness of the hands were noted. At the age of seven, examination revealed a wide based, ataxic gait. He was unable to walk heel to toe without losing his balance and was

Received for publication 15 October 1986.

Revised version accepted for publication 10 December 1986. unsteady on standing with the feet close togeth\&्छे. Muscle tone was decreased. All deep tendon feflexes were absent except for the jaw jerk, agd plantar responses were flexor. A slight degree of intention tremor on finger to nose testing was notôेd and he had difficulty performing rapid alternatigh

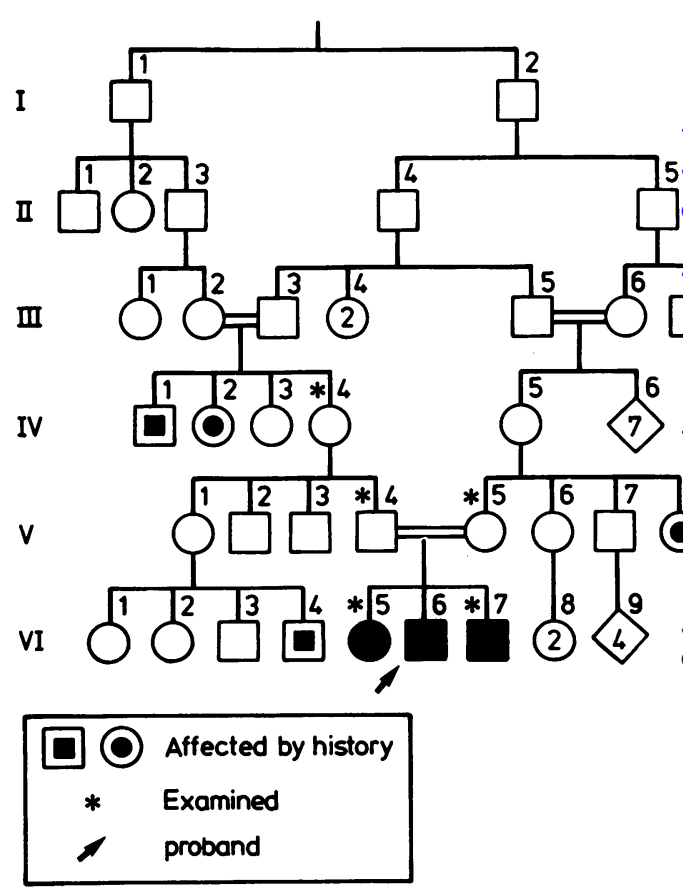

FIG 1 Family pedigree. 
movements. There was nystagmus on lateral gaze to both sides. Strength and sensation were normal. The following investigations were either normal or negative: complete blood count, glucose tolerance tests, VDRL, serum protein and lipid electrophoresis, urine screen for amino acids, chromosome analysis, routine CSF studies, $x$ rays of the thorax and spine, and CT scan. Nerve conduction studies showed an absence of sensory action potentials, with normal motor conduction in both upper and lower extremities. Electrocardiogram showed left ventricular hypertrophy and extensive $\mathrm{T}$ wave inversion.

On neurological examination at the age of 14 there was axial and appendicular ataxia which was so severe that the boy was unable to walk or stand unassisted. He had dysarthric speech and moderate ptosis of the left eyelid. There was mild thoracolumbar scoliosis, flat feet, and slight wasting of the interosseous muscles of the hands, but there was no weakness. The plantar response was extensor bilaterally. Vibration sensation was diminished up to the iliac crests and there was loss of position sense in the toes. A diastolic third heart sound was present on auscultation and a systolic ejection murmur was audible along the left sternal border. Echocardiogram showed an increase in interventricular septal thickness. There had been a progression of the previous ECG abnormalities. The patient's condition continued to deteriorate steadily and two years later he was completely wheelchair bound. His mental state was normal.

\section{Ocular findings (fig 2)}

At birth he was noted to have large eyes. The right cornea was clear, but the left showed an oedematous opacity with tears in Descemet's membrane. Intraocular pressures were $32 \mathrm{mmHg}$ OD and 45 $\mathrm{mmHg}$ OI (Schiøtz). A goniotomy was performed on the left eye. Because of the continued raised pressure in the left eye, additional goniotomies and trabeculotomy were necessary. A year later, the left eye was found to be developing first a cataract and then a spontaneous and persistent hyphema. Finally, phthisis bulbi and blindness developed in this eye. When the boy was two, the right cornea measured $14 \mathrm{~mm}$ and the left $15 \mathrm{~mm}$. The pressure in the right eye was $37 \mathrm{mmHg}$. The anterior chamber was quite deep. When viewed gonioscopically the angle was wide open, but there was increased opacification of the structures. A goniopuncture was performed on the right eye and within the next four years the patient underwent seven additional filtering procedures. Over the next years frequent examinations failed to show an increase in the intraocular pressure above $20 \mathrm{mmHg}$ in the right eye. When the boy reached 10 , a high myopia developed. The refractive error was $-12 \cdot(00$ diopters. Vision was 20/200 with myopic correction. Glaucomatous cupping of the optic disc and contraction of the visual field were noted.

\section{CASE VI.5}

The proband's 22 year old sister was normal until the age of 10 years, when progressive unsteadiness of gait with frequent falls was noticed. When she was first examined two years later, her gait was broad based and ataxic, and Romberg sign was positive. Her performance of the heel-knee-shin test was moderately impaired, with less marked incoordination on the finger-nose testing. Tone was decreased in the upper and lower limbs but there was no wasting or weakness. Deep tendon reflexes and vibratory sensation were absent in the toes. She had bilateral pes cavus with hammer toes. Routine laboratory investigations and CT scan were normal. ECG showed wide spread $T$ wave inversion. Echocardiogram was not carried out. Electrodiagnostic studies showed normal conduction velocity in the
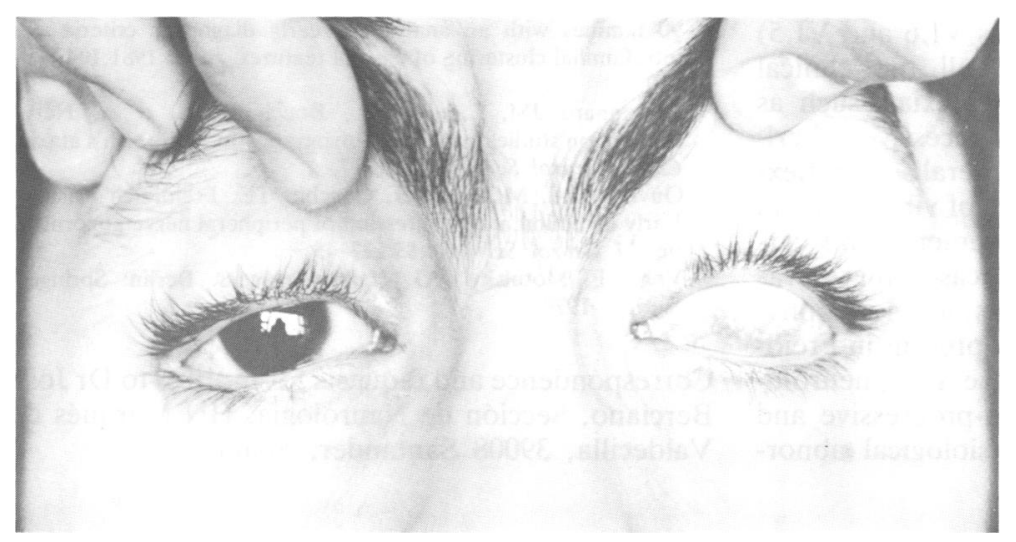

Hei 2 The eves in case VI.0.

Nole mesalocorne'a of the right oveball and phlhisis bulhi of the leli e'veball. 
motor fibres of the median and peroneal nerves, but the sensory action potentials could not be recorded.

The disease progressed slowly, forcing her to walk with a stick. At the age of 22 , examination revealed bilateral horizontal nystagmus on lateral gaze and mild dysarthria. The gait was markedly ataxic and she could not walk without support. There was mild weakness in both lower limbs and plantar responses were extensor bilaterally. Vibratory sensation and proprioception were diminished distally in all extremities, more in the legs than in the arms.

CASE VI.7

The proband's 15 year old brother was neurologically asymptomatic. At the age of five, examination revealed generalised areflexia and pes cavus. At the age of 15 , neurological examination showed the same findings together with hypopallaesthesia in the feet. Plantar responses were equivocal. There was no cerebellar ataxia. Electrophysiological studies, including motor and sensory conduction velocities of median and peroneal nerves, brainstem auditory evoked potentials, and somatosensory potentials from tibial and median nerves, were normal. No abnormalities were noted on echocardiogram and repeated ECGs.

Ocular findings in cases VI.5 and VI.7 were similar but less severe than in the proband.

\section{Discussion}

The three patients reported here showed the ocular hallmarks of congenital glaucoma. This is an unusual, inherited anomaly of the trabecular network and anterior chamber angle which leads to obstruction of aqueous outflow, increased intraocular pressure, enlarged globes (buphthalmos), corneal enlargement, oedema, and optic nerve damage. ${ }^{2}$ As in our family, autosomal recessive inheritance is considered to be the genetic basis for most cases of congenital glaucoma. ${ }^{34}$

In addition, two members (cases VI.6 and VI.5) of the affected sibship fulfilled all the clinical diagnostic criteria of Freidreich's ataxia, ${ }^{5}$ such as onset at an early age, autosomal recessive inheritance, ataxia of gait, dysarthria, generalised areflexia, extensor plantar responses, loss of vibration and position sensation, skeletal involvement, and cardiac disease. Moreover, in both cases there was absence of distal sensory potentials, an electrophysiological abnormality consistently present in Freidreich's ataxia ${ }^{67}$ By contrast, in case VI.7, neurological signs were minimal and non-progressive and there was absence of electrophysiological abnor- malities. Even though these features militate againt the actual diagnosis of Freidreich's ataxia, ${ }^{5}$ we thipek that a more prolonged follow up would be necessạy to establish whether the patient is affected or net with this form of hereditary ataxia.

The present concurrence of ataxia and congeni的 glaucoma, as described in our family, could be explained by the pleiotropic effects of one gene. Friedreich's ataxia is often accompanied by norneurological manifestations, for example, cardiac involvement, endocrine dysfunction, or skele abnormalities, and it is likely that these features aspe due to the effects of a pleiotropic gene. ${ }^{5}$ This cotfdd theoretically explain the findings in our kindred, bout to our knowledge there is no previous evidence thrdt this gene can cause congenital glaucoma. It $\mathbb{A}$ possible that more than one mutant gene is segregging in this family, especially since the likelihood of two rare mutant genes segregating together in oge family is enhanced by consanguinity. ${ }^{8}$ However, the fact that virtually all affected subjects within thiss inbred family exhibited both the neurological and ophthalmological defects might imply the transms sion of a single and new mutant recessive geqpe rather than two closely linked genes.

The authors thank the Department of Ophthalmo gy for their assistance in evaluating the patients. We would also like to thank Mrs Marta de la Fuente secretarial help and Mr John Hawkins for stylistic revision of the manuscript.

\section{References}

1 Harding AE. The hereditary ataxias and related disorders. York: Churchill Livingstone, 1984.

2 de Luise VP, Anderson DR. Primary infantile glaucoma (congenital glaucoma). Surv Ophthalmol 1983;28:1-19.

${ }^{3}$ McKusick VA. Mendelian inheritance in man. Baltimore: Jokns Hopkins University Press, 1978:515-6.

${ }^{4}$ François J. Genetic predisposition to glaucoma. Dev Opht解mol 1981;3:1-45.

5 Harding AE. Freidreich's ataxia: a clinical and genetic study? of 90 families with an analysis of early diagnostic criteria intrafamilial clustering of clinical features. Brain 1981;104:5 620.

6 Peyronnard JM, Lapointe L, Bouchard JP, et al. Ne@ve conduction studies and electromyography in Freidreich's atajia. Can J Neurol Sci 1976;3:313-7.

7 Ouvrier RA, McLeod JG, Conchin TE. Freidreich's ataks. Early detection and progression of peripheral nerve abnormatities. J Neurol Sci 1982;55:137-45.

8 Vogel F, Motulsky AG. Human genetics. Berlin: SpringrVerlag, 1979.

Correspondence and requests for reprints to Dr Jợé Berciano, Sección de Neurología, HN Marqués Valdecilla, 39008 Santander, Spain. 